\title{
Nathalie Heinich, Pourquoi Bourdieu
}

Paris, Gallimard, coll. Le débat, 2007, 192 p.

\section{Stéphane Olivesi}

\section{(2) OpenEdition}

\section{Journals}

Édition électronique

URL : http://journals.openedition.org/questionsdecommunication/1594

DOI : 10.4000/questionsdecommunication. 1594

ISSN : 2259-8901

\section{Éditeur}

Presses universitaires de Lorraine

\section{Édition imprimée}

Date de publication : 1 décembre 2008

Pagination : 368-371

ISBN : 978-2-86480-981-4

ISSN : 1633-5961

\section{Référence électronique}

Stéphane Olivesi, « Nathalie Heinich, Pourquoi Bourdieu », Questions de communication [En ligne], 14 | 2008, mis en ligne le 24 janvier 2012, consulté le 22 septembre 2020. URL : http://

journals.openedition.org/questionsdecommunication/1594 ; DOI : https://doi.org/10.4000/ questionsdecommunication.1594

Ce document a été généré automatiquement le 22 septembre 2020.

Tous droits réservés 


\title{
Nathalie Heinich, Pourquoi Bourdieu
}

\author{
Paris, Gallimard, coll. Le débat, 2007, 192 p.
}

\section{Stéphane Olivesi}

\section{RÉFÉRENCE}

Nathalie Heinich, Pourquoi Bourdieu, Paris, Gallimard, coll. Le débat, 2007, 192 p.

Pourquoi «Pourquoi Bourdieu»? C'est certainement la question que l'on se pose au premier abord et que l'on se re-pose au terme de la lecture du dernier livre de Nathalie Heinich qui se clôt sur l'évocation de la mort du propre père de l'auteure, de Jérôme Lindon et de Pierre Bourdieu. Il faut dire que, dès la première ligne, le livre tranche avec l'exégèse savante, le pamphlet ou l'hagiographie. Nathalie Heinich propose un récit à la première personne dont Pierre Bourdieu n'est souvent que le prétexte ou, pour mieux dire, une sorte de miroir. Récit à la première personne, récit presque intimiste, l'ouvrage déjoue les codes en usage non parce qu'il aurait la prétention de les pervertir, de les renouveler ou de les adapter à son objet, mais parce qu'il répond à une simple injonction de simplicité : témoigner de soi, de sa trajectoire intellectuelle, d'une vie intellectuelle à laquelle on a appartenu. Est-il donc si important de parler de Nathalie Heinich? Et - osons-le- pourquoi Heinich? Il est vrai que l'auteure a reçu l'onction extrême de Pierre Nora : seule Nathalie Heinich aurait à la fois la proximité et la distance pour parler de Pierre Bourdieu et retenir cette belle, profonde et originale problématique de recherche: comment comprendre le succès de Pierre Bourdieu? Mais Nathalie Heinich le lui rend bien. L'illustrissime Pierre Nora n'est rien moins que l'équivalent de «Heidegger, Deleuze, Foucault, Derrida, Althusser, Sartre, Aron, LéviStrauss, Ricœur, Ferry et Renaut [...] Gauchet, Furet » (p. 16) que Pierre Bourdieu aurait indistinctement critiqués. On le perçoit dès le départ: l'ouvrage ne recule ni devant l'amalgame, ni devant la confusion.

2 Premier élément d'élucidation de la problématique : Pierre Bourdieu était bel homme (p. 12)! Imagine-t-on l'une de nos modernes féministes laissait passer une quelconque allusion sur les qualités physiques d'une auteure? Et l'on sait la perversité du 
compliment : Pierre Bourdieu était un (vilain) séducteur. Il jouait de son charisme et, comme chacun le sait, le charisme, ça ne s'explique pas:"Weber avait raison: on ne l'explique pas, mais on le repère aux effets collectifs qu'il provoque ». Étrange leçon de sociologie : on ne peut pas connaitre les faits sociaux !

3 Autre élément d'élucidation : Pierre Bourdieu était "sympathique », « humain », facile d'accès avec tous ceux qui ne gravitaient pas dans son environnement professionnel direct; inversement, il «savait être redoutable avec ses concurrents». Il aurait certainement été plus productif d'analyser comment un agent, dans une conjoncture historique et scientifique très particulière, en raison d'une trajectoire sociale et intellectuelle tout aussi spécifique, parvient à occuper une position spécifique, concentrant de très nombreuses ressources, disposant d'un prestige sans égal, tout en demeurant aux marges du jeu académique. Il fallait pour cela mobiliser les outils cognitifs que Pierre Bourdieu a légués et ne pas les reléguer trop rapidement dans l'histoire des idées.

4 Il aurait d'ailleurs été intéressant d'établir un parallèle avec les trajectoires de Michel Foucault ou de Jacques Derrida, autres marginaux consacrés de la même génération, non moins "charismatiques" que pouvait l'être Pierre Bourdieu. S'inspirant de François Roustang, Nathalie Heinich préfère évoquer les sectes, sous-sectes et autres groupuscules lacaniens. Elle méconnaît un fait: Jacques Lacan fut un paria institutionnel contrairement à Pierre Bourdieu qui disposa de toutes les ressources de l'institution pour asseoir ce qu'elle désigne comme «sa domination» dans le monde intellectuel. Mais le bénéfice argumentatif de cette option n'est pas mince. On suggère que la théorie de Pierre Bourdieu fonctionne comme un délire à plusieurs. L'hypothèse est tentante. À la vérité, elle mériterait une longue enquête anthropologique car il se peut en effet que le discours théorique fonctionne en bien des cas comme le discours prophétique et que les sujets viennent s'y loger avec le confort de ceux qui habitent à peu de frais un logement luxueux et gratifiant, au risque d'oublier le monde social et d'en payer ensuite quelques sanctions. De toute évidence, Nathalie Heinich ne résiste pas à la tentation (faut-il parler à ce sujet, puisque l'on se complaît dans le registre psy, de« jouissance »?) de faire de Pierre Bourdieu un narcissique paranoïaque (pp. 32 et sq.). Le registre psy est mobilisé pour rabaisser, rabattre la singularité sur l'anormal, le pathologique. Nouvel échec de la sociologie qui s'en remet pieds et poings liés à la psychanalyse.

5 La vraie problématique de l'ouvrage, il faut donc la chercher ailleurs, plus précisément à la page 25. Nathalie Heinich a été l'étudiante de Pierre Bourdieu... Nous n'en saurons guère plus. Elle fut inscrite en thèse sous la direction de ce dernier mais Louis Marin en fut, semble- t-il, le véritable directeur... Aux initiés de l'École des hautes études en sciences sociales (EHESS) et d'ailleurs de spéculer sur les raisons du ressentiment de l'auteur, de son désamour qui s'égraine au fil de ses ouvrages dans des paragraphes flingueurs, insérés de façon impromptue entre deux développements. Toujours est-il que Nathalie Heinich, qui ne s'exprime sur Pierre Bourdieu qu'avec le regard de la Science (peut-être est-ce une nouvelle incarnation de Pierre Nora?), se confronte aux " disciples passés à la prêtrise ».

6 Il y a aussi quelques maladresses ou quelques facilités à parler du cercle initiatique pour y englober Abdelmalek Sayad, Jean-Claude Passeron, Luc Boltanski, Robert Castel, Jean- Claude Chamboredon, Alain Darbel, Dominique Schnapper (p. 23). La stigmatisation du disciple, ânonnant la voix du maître, qu'entreprend Nathalie Heinich 
peut valoir pour tous les phénomènes de cour qui ne sont pas propres à l'EHESS ou au Collège de France. Ne les observe-t-on pas sur les campus de province, autour de petits chefaillons jouant des logiques mandarinales de concentration des maigres ressources académiques, arbitrairement redistribuées, et de la mystification par l'autorité que confèrent les oripeaux de la science ? Cela dit, l'analyse de Nathalie Heinich méconnaît, minimise et occulte trois données essentielles: l'extraordinaire et très singulière expérience de travail intellectuel collectif qui se joua autour et avec Pierre Bourdieu dans une conjoncture historique et sociale particulière ; la qualité des "partenaires " de jeu de Pierre Bourdieu qui ne furent jamais de simples disciples " aveuglés " par le maitre ; les oppositions, divergences, discordances et, parfois - voire souvent - conflits, qui s'ensuivirent, témoignant d'un moment de la vie intellectuelle.

7 On s'agacera à coup sûr de la grossièreté des schémas mobilisés : les relations à l'EHESS et ailleurs se résument-elles à la dialectique du maître et du disciple ? En effet, il aurait fallu mobiliser un tout autre cadre d'analyse pour rendre compte d'une réalité caricaturée à l'extrême. On s'agacera aussi d'assertions pour le moins douteuses comme celle qui, en début d'ouvrage, nous apprend que l'on ne connaissait guère Pierre Bourdieu avant la parution de La Distinction (p. 9). Ou plus cocasse que Nathalie Heinich a, quant à elle, compris ce que Pierre Bourdieu n'était jamais parvenu à comprendre ( $p$. 175).

8 Pour finir, le chapitre 5 offre une affligeante (re-)lecture des méchants structuralistes dont fait partie Pierre Bourdieu à côté de Michel Foucault, Jacques Derrida, Jacques Lacan, héritiers intempérants de Karl Marx, Friedrich Nietzsche, Sigmund Freud auxquels est adjoint Mar tin Heidegger sans plus de justification. Elle se fonde sur les thèses de Marcel Gauchet ainsi que celles de Luc Ferry et Alain Renaut. Bref, ce qu'il s'agit de dénoncer, c'est une nouvelle fois « la pensée 68 » dont Pierre Bourdieu serait le parangon. On songe à la réaction de Michel Foucault confronté à ces journalistes en mal de vocabulaire qui l'avaient catégorisé «structuraliste » alors que point n'est besoin d'être spécialiste de son œuvre pour percevoir qu'il n'y est strictement jamais question de structure. Mais peu importe, l'essentiel pour Nathalie Heinich est de condamner tout ce qui pourrait reproduire des éléments de critique marxiste, freudien, voire nietzschéen. Dès lors, le niveau de généralité interdit que l'on puisse discuter sur le fond les propositions avancées qui amalgament quelques poncifs d'une philosophie très «science po ». Et l'on peut s'interroger : au-delà de Pierre Bourdieu, n'est-ce pas tout simplement la sociologie que récuse Nathalie Heinich au profit de l'essai quand elle rejette l'objectivation, la mise au jour de régularités statistiques, l'explication des faits par l'identification de déterminations, etc. ? Cet ultime chapitre n'apportera d'ailleurs rien au lecteur convaincu d'avance par les grands penseurs que sont Luc Ferry et Alain Renaut. Pour eux, comme pour Nathalie Heinich, Michel Foucault «s'y connaissait en dispositifs pervers» (p. 166) et Pierre Bourdieu, «théologien dogmatique » (p. 163), était un paranoïaque !

9 Parfois, trop rarement, le ressentiment parvient à être sublimé. Nathalie Heinich atteint alors son but car elle connaît son sujet et, surtout, ses faiblesses. C'est ce qui fait sa force et le seul intérêt du livre. Elle souligne à juste titre les alliances tactiques et les revirements subis dont Pierre Bourdieu fut coutumier et qui laissèrent plus d'un sans voix. Stigmatisant avec Jean-Claude Passeron («Sociologue des mythologies et mythologie de sociologues ", Les Temps modernes, 211, 1963) ceux qui discouraient sur la société de masse et les médias, Pierre Bourdieu commit Sur la télévision. Suivi de 
L'emprise du journalisme (Paris, Éd. Raisons d'agir, 1996) qui incarnait de manière exemplaire tout ce qu'il avait lui-même antérieurement combattu et dénoncé. Le plus étonnant réside certainement dans la porosité à l'égard d'enjeux profanes dont ses derniers travaux pâtissent. Ainsi tel journaliste dont le commerce présentait un médiocre intérêt conjoncturel, a-t-il pu être cité et glorifié pour être voué dès le lendemain aux gémonies sans que l'on puisse bien comprendre les raisons de ces revirements opportunistes. Nathalie Heinich épingle cette logique récurrente quand elle écrit à propos des explications que Pierre Bourdieu livra au quotidien Le Matin pour justifier son passage dans l'émission honnie "Apostrophe" lors de la sortie de La Distinction : « qu'il ne voulait pas y aller mais qu'il ne pouvait pas ne pas y aller, que tout cela était si compliqué, qu'il ne pouvait pas fâcher son éditeur ». On n'évoquera donc pas une nouvelle fois l'épisode «Abbé Pierre »... Beaucoup de choses auront opposé le savant des années 60 et 70 pratiquant de manière intégriste l'autonomie scientifique, se détournant de tout enjeu profane, et le professeur au Collège de France multipliant les interventions publiques avec plus ou moins de bonheur et, souvent, peu de rigueur. D'ailleurs, les registres savants et politiques finiront par se confondre dans ses derniers ouvrages au point d'interroger le statut de ses livres, ni réellement scientifiques, ni purement militants. On se gardera là encore de reproduire les prises de position alambiquées sur telle ou telle question de société (pp. 77-82) que Nathalie Heinich se plaît à épingler comme pour mieux souligner le peu de crédibilité de l'intellectuel.

Autre mérite de l'ouvrage : mettre en lumière certaines contradictions ou tensions dans l'œuvre telle que celle consistant à affirmer et réaffirmer le principe de neutralité axiologique, dogme incontournable que les autres chercheurs auraient trop souvent tendance à oublier, tout en s'engageant dans des luttes et en érigeant la sociologie en sport de combat (pp. 70-71). Nathalie Heinich évoque un passage du film de Pierre Carles dans lequel Pierre Bourdieu, en compagnie de collaborateurs, recherche avec quelques difficultés des données statistiques susceptibles d'être utilisées pour critiquer le néo-libéralisme. Elle ajoute avec perfidie et pertinence : «Pour un non-sociologue, cette image a toutes les chances de certifier la scientificité de la démarche : ce pourquoi sans doute le réalisateur, loin de couper cette séquence au montage, lui a donné une bonne place dans son film; mais pour un sociologue, elle est une assez cocasse caricature de travail scientifique, révélant la cuisine du tripatouillage des données lorsqu'on cherche à les utiliser non pour produire du savoir, mais pour cautionner un discours préexistant» (p. 72).

La rhétorique bourdieusienne séduit par sa force suggestive et sa fécondité heuristique; elle agace aussi. En particulier quand elle reproduit invariablement un même schéma dialectique qui consiste à identifier deux positions extrêmes opposées (structuralisme/existentialisme ; indi- vidualisme/holisme; subjectivisme/ objectivisme; empirisme/idéalisme), à associer à ces deux positions quelques œuvres pour surgir ensuite déguisé en Zorro déjouant les écueils de l'un et les pièges de l'autre et, ainsi, triompher de la vérité à bon compte. Elle agace d'autant plus que le procédé est répété, reproduit de livre en livre, éculé.

Toujours est-il que Nathalie Heinich offre un témoignage intéressant de la vie intellectuelle, de sa (petite) grandeur et de sa (grande) misère. De cette activité humaine que l'on voudrait sur-humaine. Les contraintes citationnelles («citer Bourdieu », "ne pas citer Latour ", si l'on veut bénéficier de la bienveillance de...) illustrent l'emprise des logiques sociales, des jeux d'influence dans ces univers confinés 
que sont les espaces disciplinaires où le dogmatisme règne en maître (p. 28). Et pour cette raison, parmi d'autres, n'eut-il pas mieux valu opter soit pour un travail d'exégèse plus traditionnel (qu'est-ce que Pierre Bourdieu a apporté en tel ou tel chantier de la recherche sociologique, par exemple dans celui de la sociologie de l'art et de la culture puisque c'est la spécialité de l'auteure ?), soit pour une objectivation de l'agent social «Pierre Bourdieu » afin de comprendre ce qui en fit l'extrême singularité (puisque c'est aussi un thème de réflexion développé par Nathalie Heinich) ? Ce qui intéresse l'auteure, ce n'est malheureusement pas la pensée de Pierre Bourdieu, sa validité, ses apports à la connaissance, mais le fait qu'elle a eu à ses yeux les traits et l'attrait de la science (pp.44-45), comme s'il était possible de rendre compte du phénomène en ne cherchant qu'à élucider ses modes de manifestation, en méconnaissant à dessein le fait que Pierre Bourdieu a existé scientifiquement.

\section{AUTEURS}

\section{STÉPHANE OLIVESI}

Université Lyon 2, Stephane.olivesi@univ-lyon2.fr 